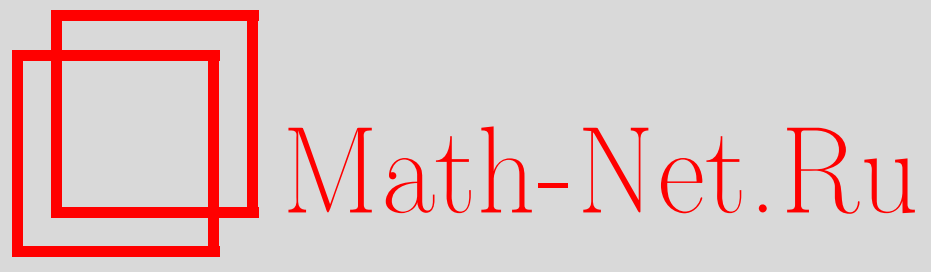

А. К. Рамазанов, Одно замечание о полиномиальных аппроксимациях с интерполяцией непрерывных функций, Матем. заметки, 2011, том 90, выпуск 2, 316-317

DOI: https://doi.org/10.4213/mzm8875

Использование Общероссийского математического портала Math-Net.Ru подразумевает, что вы прочитали и согласны с пользовательским соглашением http://www . mathnet.ru/rus/agreement

Параметры загрузки:

IP: 52.6 .47 .48

26 апреля 2023 г., 10:24:41

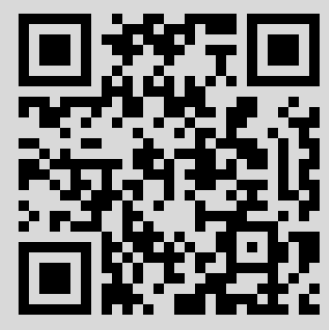




\section{Одно замечание о полиномиальных аппроксимациях с интерполяцией непрерывных функций}

\section{А. К. Рамазанов}

Как обычно, через $C[a ; b]$ будем обозначать пространство непрерывных на отрезке $[a ; b]$ функций $f$ с нормой

$$
\|f\|=\max \{|f(x)|: x \in[a ; b]\} .
$$

Зависимость скорости полиномиального приближения непрерывной функции от положения точки на данном отрезке первым исследовал Никольский [1]. Дальнейшему изучению этого вопроса посвящен ряд работ (см., например, [2]-[14]).

Теляковский [6] доказал, что если функция $f(x)$ непрерывна на данном отрезке $[a ; b]$, то для любого натурального $n$ найдется алгебраический многочлен $P_{n}(x)$ степени не выше $n$ такой, что

$$
\left|f(x)-P_{n}(x)\right| \leqslant C \omega\left(f ; \frac{\sqrt{(x-a)(b-x)}}{n}\right), \quad x \in[a ; b],
$$

где $C$ не зависит от $f, x$ и $n$, но зависит от отрезка $[a ; b]$. Здесь, как обычно, при $\delta \geqslant 0$

$$
\omega(f ; \delta)=\sup \{|f(x)-f(y)|:|x-y| \leqslant \delta ; x, y \in[a ; b]\} .
$$

Заметим, что полином $P_{n}(x)$ из неравенства (1) интерполирует приближаемую функцию $f(x)$ на концах отрезка $[a ; b]$.

Возникает естественный вопрос: справедливо ли неравенство типа Теляковского

$$
\left|f(x)-P_{n}(x)\right| \leqslant C \omega\left(f ; \frac{(x-a)^{\gamma}(b-x)^{\gamma}}{n}\right), \quad x \in[a ; b],
$$

при значении $\gamma \in(1 / 2 ; 1]$ (для любой непрерывной на отрезке $[a ; b]$ функции $f)$ ?

Ниже мы показываем, что ответ на этот вопрос в случае $1 / 2<\gamma \leqslant 1$ (как и в очевидном случае $\gamma>1$ ) отрицательный. Для построения соответствующего примера вместо отрезка $[a ; b]$ будем рассматривать отрезок $[0 ; 1]$.

Точнее, при каждом значении $\gamma \in(1 / 2 ; 1]$ существует такая непрерывная на отрезке $[0 ; 1]$ функция $f(x)$, что при всех достаточно больших натуральных $n$ найдутся точки $x \in[0 ; 1]$, в которых неравенство

$$
\left|f(x)-P_{n}(x)\right| \leqslant C \omega\left(f ; \frac{x^{\gamma}(1-x)^{\gamma}}{n}\right)
$$

не может выполняться для алгебраических полиномов $P_{n}(x)$ степени не выше $n(C$ не зависит от $f, x$ и $n$ ).

В самом деле, для значения $\gamma=1$ возьмем действительное число $k>2$, а для значения $1 / 2<\gamma<1$ - действительное число $k$, удовлетворяющее неравенству $2<k<1 /(1-\gamma)$, и рассмотрим функцию $f(x)=x^{\alpha}(x \in[0 ; 1])$ с показателем $\alpha \in(0 ;(k-2) / k)$.

Допустим, что неравенство (2) справедливо для этой функции. Тогда из неравенства (2) следует, что

$$
\left\|P_{n}\right\|-\|f\| \leqslant C \cdot 2\|f\|, \quad\left\|P_{n}\right\| \leqslant(2 C+1)\|f\|=2 C+1 .
$$

Значит, с учетом неравенства А. А. Маркова получим

$$
\left\|P_{n}^{\prime}\right\| \leqslant 2 n^{2} \cdot\left\|P_{n}\right\| \leqslant(4 C+2) n^{2} .
$$

Работа выполнена при поддержке Российского фонда фундаментальных исследований (грант № 08-01-00648a).

(C) A. K. Рамазанов, 2011 
Из наравенства (2) имеем также

$$
\left|f(x)-P_{n}(x)\right| \leqslant C \omega\left(f ; \frac{x^{\gamma}}{n}\right), \quad x \in[0 ; 1] .
$$

Так как $f(0)=P(0)=0$, применив теорему Лагранжа о среднем, из последнего неравенства получаем

$$
f(x) \leqslant\left|P_{n}^{\prime}(\zeta)\right| \cdot x+C \omega\left(f ; \frac{x^{\gamma}}{n}\right), \quad x \in[0 ; 1] .
$$

При $0<\alpha<1$ для функции $f(x)=x^{\alpha}, x \in[0 ; 1]$, имеем $\omega(f, \delta)=f(\delta)=\delta^{\alpha}$. Поэтому из неравенства (3) следует

$$
f(x) \leqslant(4 C+2) n^{2} \cdot x+C f\left(\frac{x^{\gamma}}{n}\right), \quad x \in[0 ; 1] .
$$

В частности, при $x=1 / n^{k}$ и выбранном выше $k$ отсюда получим

$$
\frac{1}{n^{k \alpha}} \leqslant \frac{4 C+2}{n^{k-2}}+\frac{C}{n^{\alpha+k \alpha \gamma}} .
$$

Это неравенство приводит к противоречию при всех достаточно больших $n$, так как параметры $k$ и $\alpha$ по их выбору удовлетворяют одновременно неравенствам

$$
k \alpha<k-2 \quad \text { и } \quad k \alpha<\alpha+k \alpha \gamma,
$$

a $C$ не зависит от $n$.

\section{СПИСОК ЦИТИРОВАННОЙ ЛИТЕРАТУРЫ}

[1] С. М. Никольский, Изв. АН СССР. Сер. матем., 10:4 (1946), 295-322. [2] А. Ф. Тиман, Докл. АН СССР, 78:1 (1951), 17-20. [3] В. К. Дзядык, Изв. АН СССР. Сер. матем., 22:3 (1958), 337-354. [4] G. Freud, Math. Ann., 137:1 (1959), 17-25. [5] Ю. А. Брудный, Докл. АН СССР, 148:6 (1963), 1237-1240. [6] С. А. Теляковский, Матем. сб., 70:2 (1966), 252-265. [7] И. Е. Гопенгауз, Матем. заметки, 1:2 (1967), 163-172. [8] R. A. DeVore, Teoрия приближения функций (Калуга, 1975), Наука, M., 1977. [9] R. Dahlhaus, J. Approx. Theory, 57:3 (1989), 274-277. [10] I. E. Gopengauz, J. Approx. Theory, 77:1 (1994), 31-41. [11] K. Kopotun, Constr. Approx., 12:1 (1996), 67-94. [12] Yu. Brudnyi, New Results in Operator Theory and its Applications, Oper. Theory Adv. Appl., 98, Birkhäuser, Basel, 1997, 92-101. [13] Р. М. Тригуб, Докл. РАН, 386:5 (2002), 599-601. [14] А.К. Рамазанов, A.-P. K. Рамазанов, Anal. Math., 35:3 (2009), 213-232.

\section{А. К. Рамазанов}

Поступило

Калужский филиал МГТУ им. Н. Э. Баумана

20.05 .2010

E-mail: akramazanov@mail.ru

Исправленный вариант

24.03.2011 